\title{
Behaviour of Broiler Birds Reared Under Coloured Light Emitting Diodes Vis-A-Vis Incandescent Light Supplemental Lighting Programme
}

\author{
Suresh Kumar ${ }^{1 *}$, Ravi Kant Gupta ${ }^{1}$, Yashpal Singh ${ }^{1}$, \\ Amit Sharma $^{1}$ and Neeraj Kashyap ${ }^{2}$ \\ ${ }^{1}$ Department of Livestock Production Management, GADVASU, Punjab, India \\ ${ }^{2}$ Department of Animal genetics and Breeding, GADVASU, Punjab, India \\ *Corresponding author
}

Key w o r d s
Broiler birds,
Incandescent bulb,
LEDs,
Supplemental light,
welfare

A B S T R A C T

The present study was conducted to assess the behaviour of broiler birds in an open-sided house under supplemental lighting programme using light emitting diodes (LEDs) and incandescent light. To perform this study a total of 240 straight run commercial Vencobb broiler chicks were procured, distributed in four different light treatment groups i.e. $\mathrm{T}_{\mathrm{w}}$; white $(650 \mathrm{~nm}), \mathrm{T}_{\mathrm{g}}$; green $(565 \mathrm{~nm})$ and $\mathrm{T}_{\mathrm{b}}$; blue $(430 \mathrm{~nm})$ light LED $(3 \mathrm{Watt}$ each) and incandescent light bulbs (60 Watt each) as control (Tc) in 12 pens (4x3 factorial design) and reared from day old to $6^{\text {th }}$ week of age. The coloured curtains were placed according to the treatment groups requirement inside each pen of shed and each pen was completely enclosed to make it light proof. Light intensity was measured with the help of light intensity meter (Lutron ${ }^{\circledR}$ PLX-111 light meter with range 0-20000 LUX). Frequency and percent duration of feeding, drinking and dozing behaviour differ significantly among different treatment groups in different weeks of study whereas, other behavioural parameters like resting, preening, wing flapping, head and litter stretching, pecking, avoiding, pushing, dust bathing, leg stretching, neck stretching and threats didn't differ significantly among different treatment groups in different weeks of study. From the study, it can be concluded that birds under LED light treatment are having significant $(p \leq 0.05)$ higher frequency and duration of feeding thus LED light bulbs could be a better alternative light source than incandescent light bulbs for commercial poultry facilities to reduce energy consumption and better economic gain without affecting the performance and welfare of broiler chickens.

\section{Introduction}

Poultry farming is an age old practice in India, livestock sector contributed about $4.11 \%$ to the total gross domestic product and poultry farming is one of the best options available with farmers. Nowadays, the major chunk of poultry production is occurring in intensively managed open sided broiler houses under artificial lighting programme requiring a lot of artificial environmental factors which differ from natural environment in which they were evolved and one of the crucial factors is light management. It is essential for sight, including both visual perspicacity and color discrimination [1]. Being a major 
environmental stimulus light affect bird behaviour significantly and it is well documented that broiler chicken showed 70\% of their behaviour as resting in all LEDs (white, red, green and blue) used whereas time spent on standing was higher in green light $(13.43 \pm 2.30 \%)$, followed by white $(10.46 \pm 2.21 \%)$, red $(9.34 \pm 1.92 \%)$ and blue $(6.78 \pm 1.92 \%)$, and pecking activity was higher in blue followed by white, green and red [2]. Therefore, light management in broiler production involving aspects of light intensity, wavelength and energy efficiency can be effectively explored. In India, so far no research work has been conducted related to the effect of LEDs on the performance of broiler chickens and scanty literature is available. Thus, the present study was conducted in broiler chicken on coloured LEDs lights with the objective to assess the effect of coloured LEDs on behaviour of broiler birds.

\section{Materials and Methods}

This study was conducted on 240 commercial Vencobb broiler chicks procured from $\mathrm{M} / \mathrm{s}$ Venkey's India (Ltd.) at the Poultry Research Farm of the Department of Livestock Production Management, Guru Angad Dev Veterinary and Animal Science University, Ludhiana (Latitude: 30 $54^{\prime}$ North and Longitude : $75^{\circ} 48^{\prime}$ East).

\section{Experiment details}

The day old sexed chicks were randomly distributed to 4 treatment groups each having 3 replicates. The housing treatments were $T_{1}$; white $(650 \mathrm{~nm}), \mathrm{T}_{2}$; green $(565 \mathrm{~nm})$ and $\mathrm{T}_{3}$; blue (430nm) light LED (3 Watt each) and incandescent light bulbs (60 Watt each) as control (Tc) in 12 pens (4x 3 factorial designs). At the day time, open-sided house was open from 10.00 AM to 4.00 PM and rest of the time sides of the house was covered with the black coloured tarpaulin sheet. Matching colour curtains were placed inside each pen of shed according to light treatment requirement and each pen was completely enclosed to make it light proof. Light intensity was measured by light intensity meter (Lutron ${ }^{\circledR}$ PLX-111 light meter with range 020000 LUX) and maintained at 25 Lux in first week and then reduces successively @ 5 Lux per week by increasing the height of bulbs from the bird eye level. The entire experimental period was divided into 3 phases namely starter (0-2 weeks), grower (3-4 weeks) and finisher (5-6 weeks). The starter, grower and finisher rations were formulated containing 22, 20 and $18 \%$ crude protein and 2896, 2932 and $2979 \mathrm{Kcal} \mathrm{ME} / \mathrm{Kg}$ of feed, respectively. The feed and water was available ad-libitum to chicks.

\section{Observations recorded}

The behavioural status of the birds was recorded using Sony ${ }^{\circledR}$ handy cam video recorder. For easy identification of birds various coloured inks was applied and instantaneous sampling technique was used for recording of behaviour of broiler chicks as continuous recording was not possible, usually because too much behaviour occurred too frequently. Daily 80 minutes behaviour recording between 06.00 and 07.40 (IST) was done, 20 minutes for each replicate of each light treatment starting from day one. The behaviour activities like preening, scratching, wing flapping, feeding, drinking, resting, pecking, avoiding, pushing, dust bathing, leg stretching, neck stretching and threats/flights of bird in response to various light sources were examined on nominal and ordinal scale. In nominal scale, data was recorded to determine the total birds expressed a particular behavioural activity in response to various light sources at a point of time. However, in ordinal scale average time spent by bird in a particular activity was assessed. 
Data on various biochemical parameters and behavioural study in relation to different treatments were subjected to one way analysis of variance (ANOVA) utilizing GLM procedure of SAS (SAS ${ }^{\circledR}$ 9.3) software and the difference among various treatments were examined by tukey's test.

\section{Results and Discussion}

The frequency of feeding behaviour during $1^{\text {st }}$, $2^{\text {nd }}$ and $4^{\text {th }}$ week was not statistically similar among different treatment groups. However, in $3^{\text {rd }}$ week frequency of feeding behaviour differ significantly $(\mathrm{p} \leq 0.05)$ in $\mathrm{T}_{1}(1.81 \pm 0.12)$ over $\mathrm{T}_{\mathrm{c}}(1.21 \pm 0.10)$ group and during $5^{\text {th }}$ week feeding behavior differ significantly $(\mathrm{p} \leq 0.05)$ in $\mathrm{T}_{1}(1.49 \pm 0.11), \mathrm{T}_{2}(1.41 \pm 0.10)$ and $\mathrm{T}_{3}$ $(1.45 \pm 0.10)$ group over $\mathrm{T}_{\mathrm{c}}(1.02 \pm 0.07)$ group and in $6^{\text {th }}$ week feeding behaviour differ significantly $(\mathrm{p} \leq 0.05)$ in $\mathrm{T}_{1}(1.50 \pm 0.10)$ and $\mathrm{T}_{2}$ $(1.75 \pm 0.10)$ group over $T_{c}(1.05 \pm 0.08)$ group.

The findings of present study was similar in agreement with the findings of [3] who found that under green coloured LED light birds spent less time in feeding $(17.2 \%)$ compared with those under white (21.\%).

The $\%$ duration time spent on drinking behaviour was significantly $(\mathrm{p} \leq 0.05)$ higher in $\mathrm{T}_{1}(2.15 \pm 0.32)$ and $\mathrm{T}_{\mathrm{c}}(1.86 \pm 0.28)$ groups as compared to $\mathrm{T}_{3}(1.01 \pm 0.20)$ in $2^{\text {nd }}$ week whereas in $3^{\text {rd }}$ week the $\%$ duration of time spent on drinking was significantly $(\mathrm{p} \leq 0.05)$ higher in $\mathrm{T}_{3}(1.46 \pm 0.25)$ group as compared to other groups.

It was found that there was no significant $(\mathrm{p} \leq 0.05)$ difference on $\%$ duration time spent on drinking behaviour at $1^{\text {st }}, 4^{\text {th }}, 5^{\text {th }}$ and $6^{\text {th }}$ week. Within group the \% duration time spent on drinking behaviour was non-significant $(\mathrm{p} \leq 0.05)$ in $\mathrm{T}_{\mathrm{c}}, \mathrm{T}_{2}$, and $\mathrm{T}_{3}$ groups whereas in $\mathrm{T}_{1}(2.15 \pm 0.32)$ group the $\%$ duration time spent on drinking was significantly $(\mathrm{p} \leq 0.05)$ higher in $2^{\text {nd }}$ week as compare to $3^{\text {rd }}$ and $4^{\text {th }}$ week and no significant $(\mathrm{p} \leq 0.05)$ difference was found in $1^{\text {st }}, 2^{\text {nd }}, 5^{\text {th }}$ and $6^{\text {th }}$ week. No significant $(\mathrm{p} \leq 0.05)$ difference was found on frequency as well as \% duration time spent on resting behaviour within groups in different weeks.

The present finds were statistically nonsignificant $(\mathrm{p} \leq 0.05)$ which were similar to the findings of [4] who found that birds spent $61 \%$ of their time resting in the litter at 6 weeks of age but resting was not significantly $(\mathrm{p} \leq 0.05)$ affected by light source and contrary to [2] and [5] who also found that there was no significant $(\mathrm{p} \leq 0.05)$ difference in time spent in resting under different coloured lights and broiler chickens preferred red followed by blue green and white.

The frequency of dozing behavior shown by the $\mathrm{T}_{1}(1.81 \pm 0.12)$ group was significantly $(\mathrm{p} \leq 0.05)$ higher as compared to control, $\mathrm{T}_{\mathrm{c}}$ $(1.20 \pm 0.10)$ and $T_{2}(1.26 \pm 0.13)$ in $3^{\text {rd }}$ week whereas frequency of dozing behaviour was significantly $(p \leq 0.05)$ lower in control as compared to LED groups in $5^{\text {th }}$ week and frequency was significantly $(\mathrm{p} \leq 0.05)$ higher in $\mathrm{T}_{1}(1.50 \pm 0.10)$ and $\mathrm{T}_{2}(1.75 \pm 0.10)$ group as compared to $\mathrm{T}_{3}(1.42 \pm 0.11)$ and $\mathrm{Tc}$ $(1.05 \pm 0.08)$ in $6^{\text {th }}$ week of birds life.

Frequency wise and \% duration time spent on different behavioural activities like preening, wing flapping, pecking, avoiding, pushing, dust bathing, leg stretching, neck stretching and threats were found to be statistically similar within groups in different weeks and between groups in different weeks.

The findings were similar and in accordance with the findings of [3], [6], [7] and [8] who found that there was no significant $(p \leq 0.05)$ difference for different light treatments in the time birds spent on drinking, dust bathing, preening and fighting (Table 1 and 2). 
Table.1 Frequency in showing different behavioral activities by birds under different treatments

\begin{tabular}{|c|c|c|c|c|c|c|c|}
\hline \multirow{2}{*}{$\begin{array}{c}\text { Behavioural } \\
\text { activities }\end{array}$} & \multirow[t]{2}{*}{ Tts. } & \multicolumn{6}{|c|}{ Age in weeks } \\
\hline & & 1 & 2 & 3 & 4 & 5 & 6 \\
\hline \multirow[t]{4}{*}{ Feeding } & $\mathbf{T}_{\mathbf{C}}$ & $1.51^{\mathrm{A}} \pm 0.10$ & $1.33^{\mathrm{AB}} \pm 0.10$ & $1.21^{\mathrm{bcABC}} \pm 0.10$ & $0.93^{\mathrm{CDE}} \pm 0.07$ & $1.02^{\mathrm{dBCDE}} \pm 0.07$ & $1.05^{\mathrm{cBCD}} \pm 0.08$ \\
\hline & $\mathbf{T}_{1}$ & $1.37^{\mathrm{BCD}} \pm 0.10$ & $1.22^{\mathrm{BCDE}} \pm 0.10$ & $1.81^{\mathrm{aA}} \pm 0.12$ & $1.22^{\mathrm{BCDE}} \pm 0.10$ & $1.49^{\mathrm{aABC}} \pm 0.11$ & $1.50^{\mathrm{bAB}} \pm 0.10$ \\
\hline & $\mathbf{T}_{2}$ & $1.45^{\mathrm{AB}} \pm 0.09$ & $1.20^{\mathrm{BCDE}} \pm 0.11$ & $1.26^{\mathrm{bcBD}} \pm 0.13$ & $1.25^{\mathrm{BCDE}} \pm 0.12$ & $1.41^{\mathrm{ac} A B C} \pm 0.10$ & $1.75^{\mathrm{abA}} \pm 0.10$ \\
\hline & $\mathbf{T}_{\mathbf{3}}$ & $1.47 \pm 0.09$ & $1.42 \pm 0.12$ & $1.53^{\mathrm{abc}} \pm 0.12$ & $1.15 \pm 0.11$ & $1.45^{\mathrm{abc}} \pm 0.10$ & $1.42^{\mathrm{abc}} \pm 0.11$ \\
\hline \multirow[t]{4}{*}{ Drinking } & $\mathbf{T}_{\mathbf{C}}$ & $0.30 \pm 0.04$ & $0.40 \pm 0.06$ & $0.17 \pm 0.04$ & $0.30 \pm 0.05$ & $0.45 \pm 0.06$ & $0.32 \pm 0.06$ \\
\hline & $\mathbf{T}_{1}$ & $0.27 \pm 0.04$ & $0.41 \pm 0.05$ & $0.17 \pm 0.04$ & $0.15 \pm 0.04$ & $0.43 \pm 0.06$ & $0.48 \pm 0.07$ \\
\hline & $\mathbf{T}_{2}$ & $0.30 \pm 0.05$ & $0.25 \pm 0.05$ & $0.21 \pm 0.05$ & $0.15 \pm 0.04$ & $0.29 \pm 0.05$ & $0.43 \pm 0.06$ \\
\hline & $\mathbf{T}_{3}$ & $0.25 \pm 0.04$ & $0.23 \pm 0.04$ & $0.34 \pm 0.05$ & $0.23 \pm 0.04$ & $0.38 \pm 0.06$ & $0.25 \pm 0.05$ \\
\hline \multirow[t]{4}{*}{ Resting } & $\mathbf{T}_{\mathbf{C}}$ & $1.89 \pm 0.07$ & $1.78 \pm 0.08$ & $1.74 \pm 0.07$ & $1.84 \pm 0.08$ & $1.89 \pm 0.08$ & $1.85 \pm 0.09$ \\
\hline & $\mathbf{T}_{1}$ & $1.86 \pm 0.07$ & $1.70 \pm 0.07$ & $1.94 \pm 0.08$ & $1.80 \pm 0.08$ & $1.82 \pm 0.08$ & $1.99 \pm 0.09$ \\
\hline & $\mathbf{T}_{2}$ & $1.80 \pm 0.07$ & $1.74 \pm 0.08$ & $1.89 \pm 0.07$ & $1.99 \pm 0.09$ & $1.84 \pm 0.08$ & $1.89 \pm 0.08$ \\
\hline & $\mathbf{T}_{3}$ & $1.95 \pm 0.08$ & $1.90 \pm 0.07$ & $1.96 \pm 0.07$ & $1.94 \pm 0.09$ & $1.71 \pm 0.07$ & $1.99 \pm 0.08$ \\
\hline \multirow[t]{4}{*}{ Dozing } & $\mathbf{T}_{\mathbf{C}}$ & $1.51^{\mathrm{A}} \pm 0.10$ & $1.33^{\mathrm{AB}} \pm 0.10$ & $1.20^{\mathrm{bcABC}} \pm 0.10$ & $0.93^{\mathrm{CDE}} \pm 0.07$ & $1.02^{\mathrm{dBCDE}} \pm 0.07$ & $1.05^{\mathrm{cBCD}} \pm 0.08$ \\
\hline & $\mathbf{T}_{1}$ & $1.37^{\mathrm{BCD}} \pm 0.10$ & $1.22^{\mathrm{BCDE}} \pm 0.10$ & $1.81^{\mathrm{aA}} \pm 0.12$ & $1.22^{\mathrm{BCDE}} \pm 0.10$ & $1.50^{\mathrm{aAB}} \pm 0.11$ & $1.50^{\mathrm{bABC}} \pm 0.10$ \\
\hline & $\mathbf{T}_{2}$ & $1.45 \pm 0.09$ & $1.20 \pm 0.11$ & $1.26^{\mathrm{b}} \pm 0.13$ & $1.25 \pm 0.12$ & $1.41^{\mathrm{ac}} \pm 0.10$ & $1.75^{\mathrm{ab}} \pm 0.10$ \\
\hline & $\mathbf{T}_{3}$ & $1.47 \pm 0.09$ & $1.42 \pm 0.12$ & $1.54^{\mathrm{abc}} \pm 0.12$ & $1.15 \pm 0.11$ & $1.45^{\mathrm{ab}} \pm 0.10$ & $1.42^{\mathrm{abc}} \pm 0.11$ \\
\hline \multirow[t]{4}{*}{ Preening } & $\mathbf{T}_{\mathbf{C}}$ & $0.09 \pm 0.02$ & $0.10 \pm 0.02$ & $0.10 \pm 0.02$ & $0.10 \pm 0.02$ & $0.08 \pm 0.02$ & $0.10 \pm 0.03$ \\
\hline & $\mathbf{T}_{1}$ & $0.09 \pm 0.02$ & $0.11 \pm 0.03$ & $0.10 \pm 0.02$ & $0.16 \pm 0.03$ & $0.11 \pm 0.03$ & $0.11 \pm 0.03$ \\
\hline & $\mathbf{T}_{2}$ & $0.07 \pm 0.02$ & $0.11 \pm 0.03$ & $0.12 \pm 0.03$ & $0.11 \pm 0.03$ & $0.08 \pm 0.02$ & $0.09 \pm 0.02$ \\
\hline & $\mathbf{T}_{\mathbf{3}}$ & $0.10 \pm 0.02$ & $0.15 \pm 0.03$ & $0.08 \pm 0.02$ & $0.11 \pm 0.02$ & $0.10 \pm 0.02$ & $0.08 \pm 0.02$ \\
\hline \multirow[t]{4}{*}{ Wing flapping } & $\mathbf{T}_{\mathbf{C}}$ & $0.12 \pm 0.02$ & $0.18 \pm 0.03$ & $0.10 \pm 0.02$ & $0.21 \pm 0.03$ & $0.16 \pm 0.03$ & $0.16 \pm 0.03$ \\
\hline & $\mathbf{T}_{1}$ & $0.10 \pm 0.02$ & $0.16 \pm 0.03$ & $0.10 \pm 0.02$ & $0.20 \pm 0.03$ & $0.11 \pm 0.03$ & $0.23 \pm 0.04$ \\
\hline & $\mathbf{T}_{2}$ & $0.11 \pm 0.02$ & $0.22 \pm 0.03$ & $0.10 \pm 0.02$ & $0.16 \pm 0.03$ & $0.12 \pm 0.03$ & $0.21 \pm 0.03$ \\
\hline & $\mathbf{T}_{\mathbf{3}}$ & $0.11 \pm 0.02$ & $0.23 \pm 0.03$ & $0.11 \pm 0.02$ & $0.17 \pm 0.03$ & $0.07 \pm 0.02$ & $0.15 \pm 0.03$ \\
\hline \multirow{3}{*}{$\begin{array}{l}\text { Head and } \\
\text { litter } \\
\text { scratching }\end{array}$} & $\mathbf{T}_{\mathbf{C}}$ & $0.17 \pm 0.03$ & $0.21 \pm 0.03$ & $0.12 \pm 0.03$ & $0.17 \pm 0.03$ & $0.10 \pm 0.02$ & $0.17 \pm 0.03$ \\
\hline & $\mathbf{T}_{1}$ & $0.12 \pm 0.02$ & $0.21 \pm 0.03$ & $0.15 \pm 0.03$ & $0.22 \pm 0.03$ & $0.19 \pm 0.03$ & $0.25 \pm 0.04$ \\
\hline & $\mathbf{T}_{2}$ & $0.15 \pm 0.03$ & $0.16 \pm 0.03$ & $0.16 \pm 0.03$ & $0.21 \pm 0.03$ & $0.14 \pm 0.03$ & $0.16 \pm 0.03$ \\
\hline
\end{tabular}




\begin{tabular}{|c|c|c|c|c|c|c|c|}
\hline & $\mathbf{T}_{\mathbf{3}}$ & $0.12 \pm 0.02$ & $0.22 \pm 0.03$ & $0.13 \pm 0.03$ & $0.22 \pm 0.03$ & $0.11 \pm 0.03$ & $0.19 \pm 0.03$ \\
\hline \multirow[t]{4}{*}{ Pecking } & $\mathbf{T}_{\mathbf{C}}$ & $0.16 \pm 0.03$ & $0.12 \pm 0.03$ & $0.08 \pm 0.02$ & $0.13 \pm 0.03$ & $0.08 \pm 0.02$ & $0.13 \pm 0.03$ \\
\hline & $\mathbf{T}_{1}$ & $0.10 \pm 0.02$ & $0.09 \pm 0.02$ & $0.13 \pm 0.03$ & $0.09 \pm 0.02$ & $0.13 \pm 0.03$ & $0.09 \pm 0.02$ \\
\hline & $\mathbf{T}_{2}$ & $0.15 \pm 0.03$ & $0.13 \pm 0.03$ & $0.12 \pm 0.03$ & $0.11 \pm 0.03$ & $0.13 \pm 0.03$ & $0.12 \pm 0.03$ \\
\hline & $\mathbf{T}_{\mathbf{3}}$ & $0.10 \pm 0.02$ & $0.09 \pm 0.02$ & $0.12 \pm 0.02$ & $0.10 \pm 0.02$ & $0.10 \pm 0.02$ & $0.08 \pm 0.02$ \\
\hline \multirow[t]{4}{*}{ Avoiding } & $\mathbf{T}_{\mathbf{C}}$ & $0.06 \pm 0.02$ & $0.09 \pm 0.02$ & $0.07 \pm 0.02$ & $0.07 \pm 0.02$ & $0.08 \pm 0.02$ & $0.06 \pm 0.02$ \\
\hline & $\mathbf{T}_{1}$ & $0.09 \pm 0.02$ & $0.08 \pm 0.02$ & $0.04 \pm 0.01$ & $0.09 \pm 0.02$ & $0.08 \pm 0.02$ & $0.07 \pm 0.02$ \\
\hline & $\mathbf{T}_{2}$ & $0.05 \pm 0.01$ & $0.08 \pm 0.02$ & $0.08 \pm 0.02$ & $0.08 \pm 0.02$ & $0.08 \pm 0.02$ & $0.08 \pm 0.02$ \\
\hline & $\mathbf{T}_{\mathbf{3}}$ & $0.09 \pm 0.02$ & $0.09 \pm 0.02$ & $0.05 \pm 0.02$ & $0.08 \pm 0.02$ & $0.03 \pm 0.01$ & $0.09 \pm 0.02$ \\
\hline \multirow[t]{4}{*}{ Pushing } & $\mathbf{T}_{\mathbf{C}}$ & $0.04 \pm 0.01$ & $0.14 \pm 0.03$ & $0.05 \pm 0.02$ & $0.13 \pm 0.03$ & $0.05 \pm 0.02$ & $0.10 \pm 0.03$ \\
\hline & $\mathbf{T}_{1}$ & $0.05 \pm 0.01$ & $0.12 \pm 0.03$ & $0.03 \pm 0.01$ & $0.13 \pm 0.03$ & $0.04 \pm 0.01$ & $0.12 \pm 0.03$ \\
\hline & $\mathbf{T}_{2}$ & $0.05 \pm 0.01$ & $0.10 \pm 0.02$ & $0.05 \pm 0.02$ & $0.13 \pm 0.03$ & $0.04 \pm 0.01$ & $0.11 \pm 0.03$ \\
\hline & $\mathbf{T}_{3}$ & $0.07 \pm 0.02$ & $0.13 \pm 0.03$ & $0.04 \pm 0.01$ & $0.13 \pm 0.03$ & $0.04 \pm 0.01$ & $0.12 \pm 0.03$ \\
\hline \multirow[t]{4}{*}{ Dust bathing } & $\mathbf{T}_{\mathbf{C}}$ & $0.10 \pm 0.02$ & $0.21 \pm 0.04$ & $0.10 \pm 0.02$ & $0.21 \pm 0.04$ & $0.08 \pm 0.02$ & $0.17 \pm 0.04$ \\
\hline & $\mathbf{T}_{1}$ & $0.13 \pm 0.02$ & $0.22 \pm 0.04$ & $0.10 \pm 0.02$ & $0.21 \pm 0.04$ & $0.13 \pm 0.03$ & $0.17 \pm 0.03$ \\
\hline & $\mathbf{T}_{2}$ & $0.07 \pm 0.02$ & $0.20 \pm 0.04$ & $0.10 \pm 0.02$ & $0.21 \pm 0.04$ & $0.09 \pm 0.02$ & $0.20 \pm 0.04$ \\
\hline & $\mathbf{T}_{3}$ & $0.12 \pm 0.02$ & $0.23 \pm 0.04$ & $0.09 \pm 0.02$ & $0.21 \pm 0.04$ & $0.13 \pm 0.03$ & $0.22 \pm 0.04$ \\
\hline \multirow[t]{4}{*}{ Leg stretching } & $\mathbf{T}_{\mathbf{C}}$ & $0.05 \pm 0.01$ & $0.05 \pm 0.02$ & $0.09 \pm 0.02$ & $0.06 \pm 0.05$ & $0.09 \pm 0.02$ & $0.08 \pm 0.02$ \\
\hline & $\mathbf{T}_{1}$ & $0.13 \pm 0.02$ & $0.08 \pm 0.02$ & $0.09 \pm 0.02$ & $0.08 \pm 0.02$ & $0.08 \pm 0.02$ & $0.04 \pm 0.01$ \\
\hline & $\mathbf{T}_{2}$ & $0.05 \pm 0.01$ & $0.08 \pm 0.02$ & $0.08 \pm 0.02$ & $0.05 \pm 0.02$ & $0.08 \pm 0.02$ & $0.08 \pm 0.02$ \\
\hline & $\mathbf{T}_{\mathbf{3}}$ & $0.13 \pm 0.02$ & $0.06 \pm 0.02$ & $0.09 \pm 0.02$ & $0.07 \pm 0.02$ & $0.10 \pm 0.02$ & $0.08 \pm 0.02$ \\
\hline \multirow{4}{*}{$\begin{array}{c}\text { Neck } \\
\text { stretching }\end{array}$} & $\mathbf{T}_{\mathbf{C}}$ & $0.14 \pm 0.02$ & $0.21 \pm 0.03$ & $0.13 \pm 0.03$ & $0.22 \pm 0.03$ & $0.14 \pm 0.03$ & $0.23 \pm 0.04$ \\
\hline & $\mathbf{T}_{1}$ & $0.11 \pm 0.02$ & $0.22 \pm 0.03$ & $0.11 \pm 0.03$ & $0.25 \pm 0.04$ & $0.13 \pm 0.03$ & $0.21 \pm 0.03$ \\
\hline & $\mathbf{T}_{2}$ & $0.12 \pm 0.02$ & $0.21 \pm 0.03$ & $0.15 \pm 0.03$ & $0.20 \pm 0.03$ & $0.12 \pm 0.03$ & $0.20 \pm 0.03$ \\
\hline & $\mathbf{T}_{\mathbf{3}}$ & $0.13 \pm 0.02$ & $0.23 \pm 0.03$ & $0.13 \pm 0.03$ & $0.22 \pm 0.03$ & $0.09 \pm 0.02$ & $0.22 \pm 0.03$ \\
\hline \multirow{4}{*}{$\begin{array}{c}\text { Threats or } \\
\text { fights }\end{array}$} & $\mathbf{T}_{\mathbf{C}}$ & $0.06 \pm 0.02$ & $0.05 \pm 0.02$ & $0.08 \pm 0.02$ & $0.05 \pm 0.02$ & $0.08 \pm 0.02$ & $0.04 \pm 0.02$ \\
\hline & $\mathbf{T}_{1}$ & $0.08 \pm 0.02$ & $0.06 \pm 0.02$ & $0.06 \pm 0.02$ & $0.03 \pm 0.01$ & $0.08 \pm 0.02$ & $0.03 \pm 0.01$ \\
\hline & $\mathbf{T}_{2}$ & $0.05 \pm 0.01$ & $0.07 \pm 0.02$ & $0.04 \pm 0.01$ & $0.05 \pm 0.02$ & $0.07 \pm 0.02$ & $0.04 \pm 0.01$ \\
\hline & $\mathbf{T}_{\mathbf{3}}$ & $0.07 \pm 0.02$ & $0.05 \pm 0.02$ & $0.05 \pm 0.02$ & $0.05 \pm 0.02$ & $0.04 \pm 0.01$ & $0.04 \pm 0.01$ \\
\hline
\end{tabular}

Means with different superscripts differ significantly $(P<0.05)$ 
Table.2 Percent duration of time spent in showing different behavioral activities by birds under different treatments

\begin{tabular}{|c|c|c|c|c|c|c|c|}
\hline \multirow{2}{*}{$\begin{array}{c}\text { Behavioural } \\
\text { activities }\end{array}$} & \multirow[t]{2}{*}{ Tts. } & \multicolumn{6}{|c|}{ Age in weeks } \\
\hline & & 1 & 2 & 3 & 4 & 5 & 6 \\
\hline \multirow[t]{4}{*}{ Feeding } & $\mathbf{T}_{\mathbf{C}}$ & $23.26^{\mathrm{A}} \pm 1.37$ & $21.62^{\mathrm{AB}} \pm 1.49$ & $18.12^{\mathrm{cBC}} \pm 1.30$ & $14.03^{\mathrm{cCDE}} \pm 1.01$ & $15.66^{\mathrm{CCDE}} \pm 1.09$ & $16.34^{\mathrm{CCD}} \pm 1.16$ \\
\hline & $\mathbf{T}_{1}$ & $21.77 \pm 1.52$ & $21.27 \pm 1.56$ & $30.37^{\mathrm{a}} \pm 1.71$ & $22.08^{\mathrm{a}} \pm 1.65$ & $24.32^{\mathrm{ab}} \pm 1.57$ & $26.02^{\mathrm{ab}} \pm 1.50$ \\
\hline & $\mathbf{T}_{2}$ & $22.67 \pm 1.34$ & $18.72 \pm 1.62$ & $20.11^{b c} \pm 1.96$ & $19.78^{\mathrm{ab}} \pm 1.79$ & $25.96^{\mathrm{a}} \pm 1.64$ & $28.48^{\mathrm{a}} \pm 1.39$ \\
\hline & $\mathbf{T}_{3}$ & $22.78 \pm 1.25$ & $20.77 \pm 1.63$ & $24.78^{\mathrm{ab}} \pm 1.71$ & $18.82^{\mathrm{abc}} \pm 1.67$ & $24.86^{\mathrm{ab}} \pm 1.48$ & $23.69^{\mathrm{ab}} \pm 1.63$ \\
\hline \multirow[t]{4}{*}{ Drinking } & $\mathbf{T}_{\mathbf{C}}$ & $1.48 \pm 0.24$ & $1.86^{\mathrm{ab}} \pm 0.28$ & $0.65^{\mathrm{b}} \pm 0.17$ & $1.43 \pm 0.27$ & $1.83 \pm 0.27$ & $1.04 \pm 0.20$ \\
\hline & $\mathbf{T}_{1}$ & $1.26^{\mathrm{ABCD}} \pm 0.21$ & $2.15^{\mathrm{A}} \pm 0.32$ & $0.66^{\mathrm{bcBCDE}} \pm 0.18$ & $0.65^{\mathrm{DE}} \pm 0.17$ & $1.76^{\mathrm{AB}} \pm 0.26$ & $1.76^{\mathrm{ABC}} \pm 0.27$ \\
\hline & $\mathbf{T}_{2}$ & $1.42 \pm 0.25$ & $0.86^{\mathrm{c}} \pm 0.18$ & $0.70^{\mathrm{b}} \pm 0.17$ & $0.66 \pm 0.19$ & $1.23 \pm 0.23$ & $1.58 \pm 0.26$ \\
\hline & $\mathbf{T}_{\mathbf{3}}$ & $1.12 \pm 0.20$ & $1.01^{\mathrm{bc}} \pm 0.20$ & $1.46^{\mathrm{a}} \pm 0.25$ & $1.10 \pm 0.22$ & $1.63 \pm 0.26$ & $0.87 \pm 0.19$ \\
\hline \multirow[t]{4}{*}{ Resting } & $\mathbf{T}_{\mathbf{C}}$ & $60.44 \pm 1.66$ & $59.38 \pm 1.59$ & $63.65^{\mathrm{ab}} \pm 1.63$ & $65.38 \pm 1.70$ & $60.50 \pm 1.80$ & $60.39 \pm 1.78$ \\
\hline & $\mathbf{T}_{1}$ & $62.55 \pm 1.69$ & $61.12 \pm 1.67$ & $53.54^{\mathrm{c}} \pm 1.69$ & $63.95 \pm 1.79$ & $58.89 \pm 1.77$ & $57.41 \pm 1.70$ \\
\hline & $\mathbf{T}_{2}$ & $58.13 \pm 1.61$ & $64.62 \pm 1.77$ & $64.77^{\mathrm{ab}} \pm 2.07$ & $64.88 \pm 1.83$ & $58.93 \pm 1.88$ & $53.48 \pm 1.56$ \\
\hline & $\mathbf{T}_{\mathbf{3}}$ & $58.46 \pm 1.50$ & $63.13 \pm 1.69$ & $60.02^{b c} \pm 1.73$ & $67.00 \pm 1.84$ & $56.80 \pm 1.65$ & $58.14 \pm 1.85$ \\
\hline \multirow[t]{4}{*}{ Dozing } & $\mathbf{T}_{\mathbf{C}}$ & $13.48^{\mathrm{abc}} \pm 0.76$ & $14.93 \pm 0.91$ & $16.66^{\mathrm{a}} \pm 0.93$ & $17.04^{\mathrm{a}} \pm 1.06$ & $21.16^{\mathrm{a}} \pm 1.22$ & $20.24^{\mathrm{a}} \pm 1.00$ \\
\hline & $\mathbf{T}_{1}$ & $13.45^{\mathrm{c}} \pm 0.90$ & $13.44 \pm 0.95$ & $14.44^{\mathrm{abc}} \pm 0.76$ & $10.99^{\text {bd }} \pm 0.76$ & $13.64^{b c} \pm 0.89$ & $12.40^{\mathrm{bc}} \pm 0.66$ \\
\hline & $\mathbf{T}_{2}$ & $16.59^{\mathrm{ab}} \pm 0.95$ & $13.54 \pm 1.00$ & $13.27^{b c} \pm 0.97$ & $12.89^{b c} \pm 1.09$ & $12.84^{b c} \pm 0.81$ & $14.30^{b c} \pm 0.81$ \\
\hline & $\mathbf{T}_{3}$ & $16.57^{b} \pm 0.76$ & $12.18 \pm 0.88$ & $12.78^{b} \pm 0.72$ & $11.07^{\mathrm{bcd}} \pm 0.66$ & $15.86^{\mathrm{b}} \pm 0.96$ & $15.21^{\mathrm{b}} \pm 0.94$ \\
\hline \multirow[t]{4}{*}{ Preening } & $\mathbf{T}_{\mathbf{C}}$ & $0.13 \pm 0.04$ & $0.31 \pm 0.09$ & $0.13 \pm 0.04$ & $0.28 \pm 0.08$ & $0.12 \pm 0.04$ & $0.25 \pm 0.07$ \\
\hline & $\mathbf{T}_{1}$ & $0.18 \pm 0.06$ & $0.28 \pm 0.07$ & $0.17 \pm 0.06$ & $0.51 \pm 0.12$ & $0.24 \pm 0.08$ & $0.29 \pm 0.09$ \\
\hline & $\mathbf{T}_{2}$ & $0.07 \pm 0.03$ & $0.34 \pm 0.10$ & $0.17 \pm 0.05$ & $0.31 \pm 0.09$ & $0.07 \pm 0.03$ & $0.29 \pm 0.10$ \\
\hline & $\mathbf{T}_{\mathbf{3}}$ & $0.22 \pm 0.07$ & $0.48 \pm 0.11$ & $0.16 \pm 0.06$ & $0.29 \pm 0.07$ & $0.20 \pm 0.07$ & $0.29 \pm 0.09$ \\
\hline \multirow{4}{*}{$\begin{array}{c}\text { Wing } \\
\text { flapping }\end{array}$} & $\mathbf{T}_{\mathbf{C}}$ & $0.17 \pm 0.04$ & $0.59 \pm 0.11$ & $0.10 \pm 0.03$ & $0.70 \pm 0.12$ & $0.11 \pm 0.03$ & $0.53 \pm 0.12$ \\
\hline & $T_{1}$ & $0.13 \pm 0.04$ & $0.48 \pm 0.11$ & $0.14 \pm 0.04$ & $0.69 \pm 0.12$ & $0.14 \pm 0.05$ & $0.79 \pm 0.13$ \\
\hline & $\mathbf{T}_{2}$ & $0.16 \pm 0.04$ & $0.64 \pm 0.12$ & $0.14 \pm 0.04$ & $0.54 \pm 0.11$ & $0.14 \pm 0.04$ & $0.70 \pm 0.12$ \\
\hline & $\mathbf{T}_{\mathbf{3}}$ & $0.13 \pm 0.04$ & $0.74 \pm 0.13$ & $0.17 \pm 0.05$ & $0.54 \pm 0.11$ & $0.07 \pm 0.03$ & $0.50 \pm 0.11$ \\
\hline \multirow{3}{*}{$\begin{array}{l}\text { Head and } \\
\text { litter } \\
\text { scratching }\end{array}$} & $\mathbf{T}_{\mathbf{C}}$ & $0.75^{\mathrm{a}} \pm 0.15$ & $0.64 \pm 0.13$ & $0.38 \pm 0.11$ & $0.54 \pm 0.12$ & $0.31 \pm 0.10$ & $0.41 \pm 0.10$ \\
\hline & $\mathbf{T}_{1}$ & $0.21^{\mathrm{c}} \pm 0.06$ & $0.67 \pm 0.13$ & $0.49 \pm 0.13$ & $0.64 \pm 0.13$ & $0.61 \pm 0.14$ & $0.79 \pm 0.15$ \\
\hline & $\mathbf{T}_{2}$ & $0.65^{\mathrm{ab}} \pm 0.14$ & $0.50 \pm 0.12$ & $0.50 \pm 0.12$ & $0.70 \pm 0.15$ & $0.46 \pm 0.12$ & $0.57 \pm 0.14$ \\
\hline
\end{tabular}




\begin{tabular}{|c|c|c|c|c|c|c|c|}
\hline & $\mathbf{T}_{\mathbf{3}}$ & $0.25^{\mathrm{bc}} \pm 0.07$ & $0.76 \pm 0.14$ & $0.45 \pm 0.12$ & $0.67 \pm 0.13$ & $0.36 \pm 0.11$ & $0.65 \pm 0.14$ \\
\hline \multirow[t]{4}{*}{ Pecking } & $\mathbf{T}_{\mathbf{C}}$ & $0.08 \pm 0.01$ & $0.15 \pm 0.05$ & $0.04 \pm 0.01$ & $0.21 \pm 0.07$ & $0.04 \pm 0.01$ & $0.30 \pm 0.09$ \\
\hline & $\mathbf{T}_{1}$ & $0.05 \pm 0.01$ & $0.17 \pm 0.07$ & $0.06 \pm 0.01$ & $0.17 \pm 0.07$ & $0.07 \pm 0.02$ & $0.11 \pm 0.05$ \\
\hline & $\mathbf{T}_{2}$ & $0.07 \pm 0.01$ & $0.25 \pm 0.08$ & $0.06 \pm 0.01$ & $0.12 \pm 0.05$ & $0.06 \pm 0.01$ & $0.12 \pm 0.05$ \\
\hline & $\mathbf{T}_{\mathbf{3}}$ & $0.05 \pm 0.01$ & $0.24 \pm 0.14$ & $0.06 \pm 0.01$ & $0.20 \pm 0.07$ & $0.05 \pm 0.01$ & $0.20 \pm 0.07$ \\
\hline \multirow[t]{4}{*}{ Avoiding } & $\mathbf{T}_{\mathbf{C}}$ & $0.02 \pm 0.00$ & $0.07 \pm 0.02$ & $0.02 \pm 0.00$ & $0.06 \pm 0.02$ & $0.03 \pm 0.01$ & $0.05 \pm 0.02$ \\
\hline & $\mathbf{T}_{1}$ & $0.03 \pm 0.01$ & $0.05 \pm 0.01$ & $0.01 \pm 0.00$ & $0.08 \pm 0.02$ & $0.05 \pm 0.02$ & $0.04 \pm 0.01$ \\
\hline & $\mathbf{T}_{2}$ & $0.01 \pm 0.00$ & $0.06 \pm 0.02$ & $0.03 \pm 0.01$ & $0.07 \pm 0.02$ & $0.03 \pm 0.01$ & $0.05 \pm 0.02$ \\
\hline & $\mathbf{T}_{\mathbf{3}}$ & $0.03 \pm 0.01$ & $0.12 \pm 0.05$ & $0.01 \pm 0.00$ & $0.06 \pm 0.02$ & $0.00 \pm 0.00$ & $0.08 \pm 0.02$ \\
\hline \multirow[t]{4}{*}{ Pushing } & $\mathbf{T}_{\mathbf{C}}$ & $0.01 \pm 0.00$ & $0.07 \pm 0.01$ & $0.01 \pm 0.00$ & $0.06 \pm 0.01$ & $0.02 \pm 0.00$ & $0.05 \pm 0.01$ \\
\hline & $T_{1}$ & $0.01 \pm 0.00$ & $0.06 \pm 0.01$ & $0.01 \pm 0.00$ & $0.06 \pm 0.01$ & $0.01 \pm 0.00$ & $0.06 \pm 0.01$ \\
\hline & $\mathbf{T}_{2}$ & $0.02 \pm 0.00$ & $0.05 \pm 0.01$ & $0.01 \pm 0.00$ & $0.06 \pm 0.01$ & $0.01 \pm 0.00$ & $0.05 \pm 0.01$ \\
\hline & $\mathbf{T}_{\mathbf{3}}$ & $0.02 \pm 0.00$ & $0.08 \pm 0.02$ & $0.01 \pm 0.00$ & $0.06 \pm 0.01$ & $0.01 \pm 0.00$ & $0.06 \pm 0.01$ \\
\hline \multirow{4}{*}{$\begin{array}{c}\text { Dust } \\
\text { bathing }\end{array}$} & $\mathbf{T}_{\mathbf{C}}$ & $0.07 \pm 0.02$ & $0.15 \pm 0.06$ & $0.09 \pm 0.02$ & $0.11 \pm 0.03$ & $0.05 \pm 0.02$ & $0.09 \pm 0.02$ \\
\hline & $\mathbf{T}_{1}$ & $0.12 \pm 0.03$ & $0.08 \pm 0.02$ & $0.08 \pm 0.02$ & $0.11 \pm 0.03$ & $0.12 \pm 0.03$ & $0.08 \pm 0.02$ \\
\hline & $\mathbf{T}_{2}$ & $0.06 \pm 0.02$ & $0.11 \pm 0.03$ & $0.10 \pm 0.03$ & $0.08 \pm 0.01$ & $0.07 \pm 0.02$ & $0.10 \pm 0.03$ \\
\hline & $\mathbf{T}_{3}$ & $0.13 \pm 0.03$ & $0.14 \pm 0.04$ & $0.09 \pm 0.03$ & $0.08 \pm 0.02$ & $0.11 \pm 0.03$ & $0.11 \pm 0.02$ \\
\hline \multirow{4}{*}{$\begin{array}{c}\text { Leg } \\
\text { stretching }\end{array}$} & $\mathbf{T}_{\mathbf{C}}$ & $0.03 \pm 0.01$ & $0.05 \pm 0.01$ & $0.04 \pm 0.01$ & $0.06 \pm 0.02$ & $0.04 \pm 0.01$ & $0.06 \pm 0.02$ \\
\hline & $T_{1}$ & $0.05 \pm 0.01$ & $0.07 \pm 0.02$ & $0.04 \pm 0.01$ & $0.08 \pm 0.02$ & $0.03 \pm 0.01$ & $0.04 \pm 0.01$ \\
\hline & $\mathbf{T}_{2}$ & $0.03 \pm 0.01$ & $0.07 \pm 0.02$ & $0.03 \pm 0.01$ & $0.04 \pm 0.01$ & $0.04 \pm 0.01$ & $0.05 \pm 0.02$ \\
\hline & $\mathbf{T}_{\mathbf{3}}$ & $0.06 \pm 0.01$ & $0.05 \pm 0.02$ & $0.04 \pm 0.01$ & $0.06 \pm 0.02$ & $0.05 \pm 0.01$ & $0.07 \pm 0.02$ \\
\hline \multirow{4}{*}{$\begin{array}{c}\text { Neck } \\
\text { stretching }\end{array}$} & $\mathbf{T}_{\mathbf{C}}$ & $0.08 \pm 0.01$ & $0.14 \pm 0.02$ & $0.07 \pm 0.01$ & $0.15 \pm 0.02$ & $0.07 \pm 0.01$ & $0.16 \pm 0.03$ \\
\hline & $\mathbf{T}_{1}$ & $0.06 \pm 0.01$ & $0.15 \pm 0.02$ & $0.06 \pm 0.01$ & $0.16 \pm 0.03$ & $0.07 \pm 0.01$ & $0.16 \pm 0.03$ \\
\hline & $\mathbf{T}_{2}$ & $0.07 \pm 0.01$ & $0.15 \pm 0.02$ & $0.08 \pm 0.01$ & $0.14 \pm 0.02$ & $0.06 \pm 0.01$ & $0.14 \pm 0.02$ \\
\hline & $\mathbf{T}_{\mathbf{3}}$ & $0.07 \pm 0.01$ & $0.18 \pm 0.03$ & $0.07 \pm 0.01$ & $0.15 \pm 0.02$ & $0.05 \pm 0.01$ & $0.14 \pm 0.02$ \\
\hline \multirow{4}{*}{$\begin{array}{l}\text { Threats or } \\
\text { fights }\end{array}$} & $\mathbf{T}_{\mathbf{C}}$ & $0.06 \pm 0.02$ & $0.04 \pm 0.01$ & $0.07 \pm 0.02$ & $0.05 \pm 0.01$ & $0.07 \pm 0.02$ & $0.03 \pm 0.01$ \\
\hline & $\mathbf{T}_{1}$ & $0.06 \pm 0.02$ & $0.04 \pm 0.02$ & $0.04 \pm 0.02$ & $0.02 \pm 0.01$ & $0.08 \pm 0.03$ & $0.02 \pm 0.01$ \\
\hline & $\mathbf{T}_{2}$ & $0.05 \pm 0.02$ & $0.06 \pm 0.02$ & $0.04 \pm 0.02$ & $0.04 \pm 0.01$ & $0.06 \pm 0.02$ & $0.02 \pm 0.01$ \\
\hline & $\mathbf{T}_{3}$ & $0.05 \pm 0.02$ & $0.05 \pm 0.02$ & $0.04 \pm 0.01$ & $0.04 \pm 0.01$ & $0.03 \pm 0.01$ & $0.03 \pm 0.01$ \\
\hline
\end{tabular}

Means with different superscripts differ significantly $(P<0.05)$ 
From this study, it can be concluded that birds under coloured light emitting diode (LED) bulbs treatment groups were having significant $(\mathrm{p} \leq 0.05)$ higher frequency and duration of feeding without affecting the overall welfare of broiler birds. The use of LED in broiler industry will definitely saves a lot of electricity and increases the return from poultry sector.

\section{Acknowledgement}

The authors are thankful to the worthy ViceChancellor, GADVASU, Ludhiana for extending necessary support to carry out this research work.

\section{References}

1. C.E. Manser, Effects of lighting on the welfare of domestic poultry: A review, Animal Welfare 5, 1996, 341-360.

2. N. Kim, S.R. Lee and S.J. Lee, Effects of light color on energy expenditure and behaviour in broiler chickens. AsianAustralian Journal of Animal Science 27(7), 2014, 1044-1049.

3. H.B. Eicher and A. Suter, Effects of colored light-emitting diode illumination on behavior and performance of laying hens. Poultry science 92, 2013, 869-73.

4. H.H. Kristensen, N.B. Prescott, G.C. Perry, The behaviour of broiler chickens in different light sources and illuminances. Applied animal behaviour science 103, 2007, 75-89

5. D.S. Prayitno, C.J.C. Phillips and H. Omed, The Effects of Color of Lighting on the Behavior and Production of Meat Chickens. Poultry Science 76, 1997, 452-57.

6. I. Rozenboim, I. Biran, Z. Uni, B. Robinzon and O. Halevy, The effect of monochromatic light on broiler growth and development. Poultry Science 78, 1998, 135-38.

7. P.D. Lewis and T.R. Morris, Responses of domestic poultry to various light sources. World's Poultry Science Journal 56, 1998, 189-207.

8. S. Firouzi, H. Nazarpak, H. Habibi and M. Marzban, Effects of color lights on performance, immune response and hematological indices of broilers. Journal of World's Poultry Research $4(2), 2014,52-55$.

\section{How to cite this article:}

Suresh Kumar, Ravi Kant Gupta, Yashpal Singh, Amit Sharma and Neeraj Kashyap. 2019. Behaviour of Broiler Birds Reared Under Coloured Light Emitting Diodes Vis-A-Vis Incandescent Light Supplemental Lighting Programme. Int.J.Curr.Microbiol.App.Sci. 8(07): 423-430. doi: https://doi.org/10.20546/ijcmas.2019.807.052 